\title{
Mesial temporal lobe epilepsy: long-term seizure outcome of patients primarily treated with transsylvian selective amygdalohippocampectomy
}

\author{
Christian Dorfer, MD, ${ }^{1}$ Thomas Czech, MD, ${ }^{1}$ Susanne Aull-Watschinger, MD, ${ }^{2}$ \\ Christoph Baumgartner, MD, ${ }^{3}$ Rebekka Jung, MD, ${ }^{2}$ Gregor Kasprian, MD, ${ }^{4}$ Klaus Novak, MD, ${ }^{1}$ \\ Susanne Pirker, MD, ${ }^{3}$ Birgit Seidl, MSc, ${ }^{2}$ Harald Stefanits, MD, ${ }^{1}$ Karin Trimmel, MD, ${ }^{2}$ and \\ Ekaterina Pataraia, MD²
}

\begin{abstract}
Departments of ${ }^{1}$ Neurosurgery, ${ }^{2}$ Neurology, and ${ }^{4}$ Radiology and Nuclear Medicine, Medical University of Vienna; and ${ }^{3}$ Karl Landsteiner Institute for Clinical Epilepsy Research and Cognitive Neurology, General Hospital Hietzing with Neurological Center Rosenhügel, Vienna, Austria
\end{abstract}

\begin{abstract}
OBJECTIVE The aim of this study was to present long-term seizure outcome data in a consecutive series of patients with refractory mesial temporal lobe epilepsy primarily treated with transsylvian selective amygdalohippocampectomy (SAHE).
\end{abstract}
METHODS The authors retrospectively analyzed prospectively collected data for all patients who had undergone resec- tive surgery for medically refractory epilepsy at their institution between July 1994 and December 2014. Seizure outcome was assessed according to the International League Against Epilepsy (ILAE) and the Engel classifications.

RESULTS The authors performed an SAHE in 158 patients (78 males, 80 females; 73 right side, 85 left side) with a mean age of $37.1 \pm 10.0$ years at surgery. Four patients lost to follow-up and 1 patient who committed suicide were excluded from analysis. The mean follow-up period was 9.7 years. At the last available follow-up (or before reoperation), 68 patients (44.4\%) had achieved an outcome classified as ILAE Class 1a, 46 patients (30.1\%) Class 1, 6 patients $(3.9 \%)$ Class 2, 16 patients (10.4\%) Class 3, 15 patients (9.8\%) Class 4, and 2 patients (1.3\%) Class 5. These outcomes correspond to Engel Class I in $78.4 \%$ of the patients, Engel Class II in 10.5\%, Engel Class III in $8.5 \%$, and Engel Class IV in $2.0 \%$. Eleven patients underwent a second surgery (anterior temporal lobectomy) after a mean of 4.4 years from the SAHE (left side in 6 patients, right side in 5). Eight (72.7\%) of these 11 patients achieved seizure freedom.

The overall ILEA seizure outcome since (re)operation after a mean follow-up of 10.0 years was Class 1a in 72 patients $(47.0 \%)$, Class 1 in 50 patients (32.6\%), Class 2 in 7 patients (4.6\%), Class 3 in 15 patients (9.8\%), Class 4 in 8 patients $(5.2 \%)$, and Class 5 in 1 patient $(0.6 \%)$. These outcomes correspond to an Engel Class I outcome in $84.3 \%$ of the patients.

CONCLUSIONS A satisfactory long-term seizure outcome following transsylvian SAHE was demonstrated in a selected group of patients with refractory temporal lobe epilepsy.

https://thejns.org/doi/abs/10.3171/2017.4.JNS162699

KEY WORDS temporal lobe epilepsy; selective amygdalohippocampectomy; temporal lobectomy

$\mathrm{T}$ EMPORAL lobe epilepsy with associated hippocampal sclerosis is the most frequent form of focal epilepsy that is refractory to medical treatment. The benefit of resective surgery has been convincingly demonstrated in multiple studies. ${ }^{14,15,57}$ While the crucial importance of including medial temporal structures in the resection has been acknowledged since the experience of the Montreal group, the question of the optimal extent of resection (selective limbic resection vs standard anterior temporal lobectomy $[\mathrm{ATL}])$ in the individual patient has not yet been resolved. . $, 6,27,31,49^{2}$

At the time that the formal epilepsy surgery program

ABBREVIATIONS AED = antiepileptic drug; ATL = anterior temporal lobectomy; EEG = electroencephalography; IED = interictal epileptiform discharge; ILAE = International League Against Epilepsy; MTLE = mesial temporal lobe epilepsy; SAHE = selective amygdalohippocampectomy.

SUBMITTED October 25, 2016. ACCEPTED April 4, 2017.

INCLUDE WHEN CITING Published online October 13, 2017; DOI: 10.3171/2017.4.JNS162699. 
was started in Vienna in 1994, the Second Palm Desert Survey documented similar seizure freedom rates between selective limbic resections (68.8\%) and ATL (67.9\%). ${ }^{15}$ These results refuted the argument that a more extensive neocortical resection is necessary to obtain good seizure control. ${ }^{15}$ Thus, it has been our strategy to tailor the temporal resection to the results of our preoperative patient investigations, and we have opted for the transsylvian technique in patients assumed to be eligible for selective amygdalohippocampectomy (SAHE) ${ }^{62}$

We present here the experience at our center in using the transsylvian SAHE in patients deemed eligible for selective resection; the same epileptological team and the same surgeon performed all resections.

\section{Methods \\ Patients}

We retrospectively reviewed prospectively collected data on 427 adults who had undergone resective surgery for medically refractory epilepsy at the Vienna Epilepsy Program between July 1994 and December 2014. Of these patients, 295 suffered from temporal lobe epilepsy not associated with structural lesions other than hippocampal sclerosis or temporal lobe atrophy. Based on the criteria outlined below, 158 were assigned to SAHE as the first surgical treatment.

The study protocol was reviewed and approved by the local ethics committee.

\section{Presurgical Evaluation}

All patients were subjected to extensive evaluation before surgery. The presurgical workup comprised neurological examination and history, prolonged video electroencephalography (EEG) monitoring, high-resolution MRI, Wada testing or functional MRI for assessment of language and memory function, formal neuropsychological testing, and visual field examination using the standard automated perimetry examination. In the majority of patients, interictal PET was performed. Video EEG monitoring was recorded for an average of 5 days; the EEG activity was recorded according to the extended international 10-20 system, including bilaterally placed true anterior temporal electrodes and sphenoidal electrodes. Seizure semiology, including clinical localizing and lateralizing signs, was evaluated with respect to predicting the side of the epileptogenic zone. ${ }^{35}$ Absolute spike frequency and location of interictal epileptiform discharges (IEDs) were assessed by visual analyses over the entire recording time. The distribution of IEDs was defined as unilateral if $>90 \%$ of the spikes occurred over the affected temporal lobe. ${ }^{7}$ Ictal scalp EEG patterns were determined by morphology, location, and time course of ictal EEG changes. ${ }^{18,43,51}$

All patients underwent high-resolution MRI, which was initially performed with a 1.5-T unit (Gyroscan ACSNT, Philips Medical Systems). Starting in 2006, MRI was performed on a 3.0-T machine. A temporal lobe protocol was applied for detailed depiction of the temporal lobe structures (guidelines for neuroimaging evaluation of patients with uncontrolled epilepsy who are being considered for surgery). ${ }^{55}$
The following variables were assessed: age at epilepsy manifestation; duration of epilepsy prior to surgery; age at operation; history of febrile seizures, meningitis/encephalitis, or traumatic brain injury as well as other medical history; type of seizures and their frequency; and peri- and postoperative surgical complications; antiepileptic drugs (AEDs) were evaluated before and after surgery.

The decision regarding the type of operation to be performed was made individually for each patient after the presurgical workup and the case discussion at a multidisciplinary epilepsy conference were completed. The criteria to perform an SAHE over an upfront ATL were as follows: 1) congruent results from video EEG monitoring pointing to mesial temporal onset (ipsilateral unitemporal IEDs, ipsilateral temporal ictal EEG patterns, and clinical lateralizing signs corresponding to the ipsilateral temporal region or nonlateralizable signs clearly corresponding to temporal lobe origin, that is, oral automatisms, behavioral arrest, and so forth), ${ }^{1,20,45}$ 2) ipsilateral hippocampal sclerosis and/or atrophy on MRI, 3) absence of unequivocal atrophy in the lateral temporal lobe, and 4) absence of any other pathology in the temporal lobe.

\section{Surgical Procedure: SAHE}

All procedures were performed by one neurosurgeon (T.C.). In all patients, the transsylvian transamygdala route developed by Yaşargil was used: ${ }^{62}$ After opening the sylvian fissure, a corticotomy on the temporal side of the limen insulae was performed. By resection and suction of the amygdala and adjoining uncal cortex up to the level of the optic tract, the anterior roof of the temporal horn was opened. After detaching the choroid plexus from its tenia fimbriae and dissecting the arterial branches to the hippocampal sulcus, subpial dissection of the medial parahippocampal gyrus was started. The posterior margin of the resection was defined by a transverse transependymal cut through the most posterior part of the hippocampus. The collateral sulcus was exposed through the ventricular wall lateral to the hippocampus. This incision was connected to the posterior transverse hippocampal incision, and the lateral limit of the resection was defined by following this sulcus rostrally, taking into account information on the individual sulcal pattern provided by MRI ${ }^{39}$ After dissecting around the hippocampal head in a subpial plane, cutting the unco-hippocampal arteries passing through the uncal sulcus, and lastly coagulating and cutting the vein usually present at the anterior end of the choroid fissure and draining into the basal vein, the parahippocampal-hippocampal specimen was removed en bloc. Finally, in every case an effort was made to resect by suction the cortex medial to the rostral prolongation of the collateral sulcus including the posterior superomedial polar cortex. The resected tissue was sent for histopathological analyses in all cases.

\section{Outcome Assessment}

Follow-up was conducted at 4 months and 1,2,5, 10, 15, and 20 years postoperatively. The face-to-face interview included neurological examination, scalp EEG, neuropsychological testing, and visual field examination. Magnetic resonance imaging was performed at 4 months after surgery. In addition, seizure outcome and current AEDs were 
evaluated yearly based on telephone interviews. Outcome was scored using the stringent classification of the International League Against Epilepsy (ILAE) ${ }^{58}$ which allows for the identification of patients who have been completely seizure free since surgery. Furthermore, we apposed the ILAE classification and the Engel classification as proposed by Wieser et al. for better comparability with the literature. ${ }^{15,59}$ Accordingly, ILAE Classes 1, 1a, and 2 are comparable to Engel Class I, ILAE Class 3 is comparable to Engel Class II, ILAE Class 4 is comparable to Engel Class III, and ILAE Classes 5 and 6 are comparable to Engel Class IV. The patients with seizures on attempted AED withdrawal were considered to be not seizure free.

The patients whose first operation failed were offered reevaluation according to the criteria described above and, if possible, reoperation. The decision regarding the type of reoperation to perform was made individually for each patient after the reevaluation and case discussion at a multidisciplinary epilepsy conference were completed. The extent of the temporal pole resection was dependent on the laterality of language and memory, with $3-4.5 \mathrm{~cm}$ on the dominant side and $4.5-5.5 \mathrm{~cm}$ on the nondominant side.

\section{Statistical Analysis}

For unifactorial analysis between groups defined by outcome, the Student t-test was used for statistical comparison of continuous prognostic variables if the distribution was normal, and the Wilcoxon test was used if the distribution was not normal. Dichotomous discrete variables were analyzed using the chi-square test or the Fisher exact test. All analyses were performed using SSPS version 18.0 (SPSS Inc.).

\section{Results}

\section{Seizure Outcome After SAHE}

We performed an SAHE in 158 patients (78 males, 80 females; 73 right side, 85 left side) with a mean age of 37.1 \pm 10.0 years (median 37.5 years, range $16.0-62.0$ years) at surgery. We excluded from further analysis the data for 4 patients $(2.5 \%)$ who were lost to follow-up and 1 patient $(0.6 \%)$ who committed suicide 4 months after surgery.

Seizure outcomes were documented up to 20 years following surgery, with a mean follow-up time of 9.7 years (median 10.0 years, range 18 months-20.1 years). At the last available follow-up (or before reoperation), complete cure of seizures since surgery (ILAE Class 1a) had been achieved in 68 patients (44.4\%). Forty-six patients (30.1\%) had been seizure free at least 12 months before the last follow-up visit (ILAE Class 1), 6 patients (3.9\%) had only auras (ILAE Class 2), and 31 patients (20.3\%) benefited from surgery, with $16(10.4 \%)$ achieving ILAE Class 3 and $15(9.8 \%)$ achieving ILAE Class 4 . Two patients (1.3\%) did not benefit from surgery and were classified as ILAE Class 5. The corresponding outcomes according to the Engel classification were Engel Class I in $78.4 \%$ of patients, Engel Class II in $10.5 \%$, Engel Class III in $8.5 \%$, and Engel Class IV in 2.0\%. No patient had an appreciable worsening of epilepsy (ILEA Class 6 or Engel Class IVC).

During the follow-up period, seizure freedom rates remained stable when applying ILAE Class 1 (that is, no sei- zures during a 12-month period prior to assessment). One hundred eight $(70.6 \%)$ of 153 patients were seizure free at 1 year, $96(64.9 \%)$ of 148 patients at 3 years, 101 (78.9\%) of 128 patients at 5 years, $80(81.6 \%)$ of 98 patients at 10 years, $46(88.5 \%)$ of 51 patients at 15 years, and $4(57.1 \%)$ of 7 patients at 20 years after SAHE. Figure 1 shows the number of patients with or without seizures at different time points after surgery and the number of patients changing between the seizure-free and non-seizure-free groups.

When analyzing our cohort using the stringent but clinically more relevant definition of seizure freedom (that is, ILAE Class 1a, patients completely seizure free since surgery), we found a smaller number of successfully treated patients and a decrease in the number of patients without recurrence of seizures and auras over time: $70.6 \%$ at 1 year, $61.3 \%$ at 2 years, $54.3 \%$ at 5 years, $50 \%$ at 10 years, $50 \%$ at 15 years, and $28.6 \%$ at 20 years (Fig. 2).

Our analysis of potential predictors of seizure outcome such as age at surgery; age at seizure onset; number of AEDs; history of traumatic brain injury, meningitis, or febrile seizures; and seizure frequency did not reveal any significant findings.
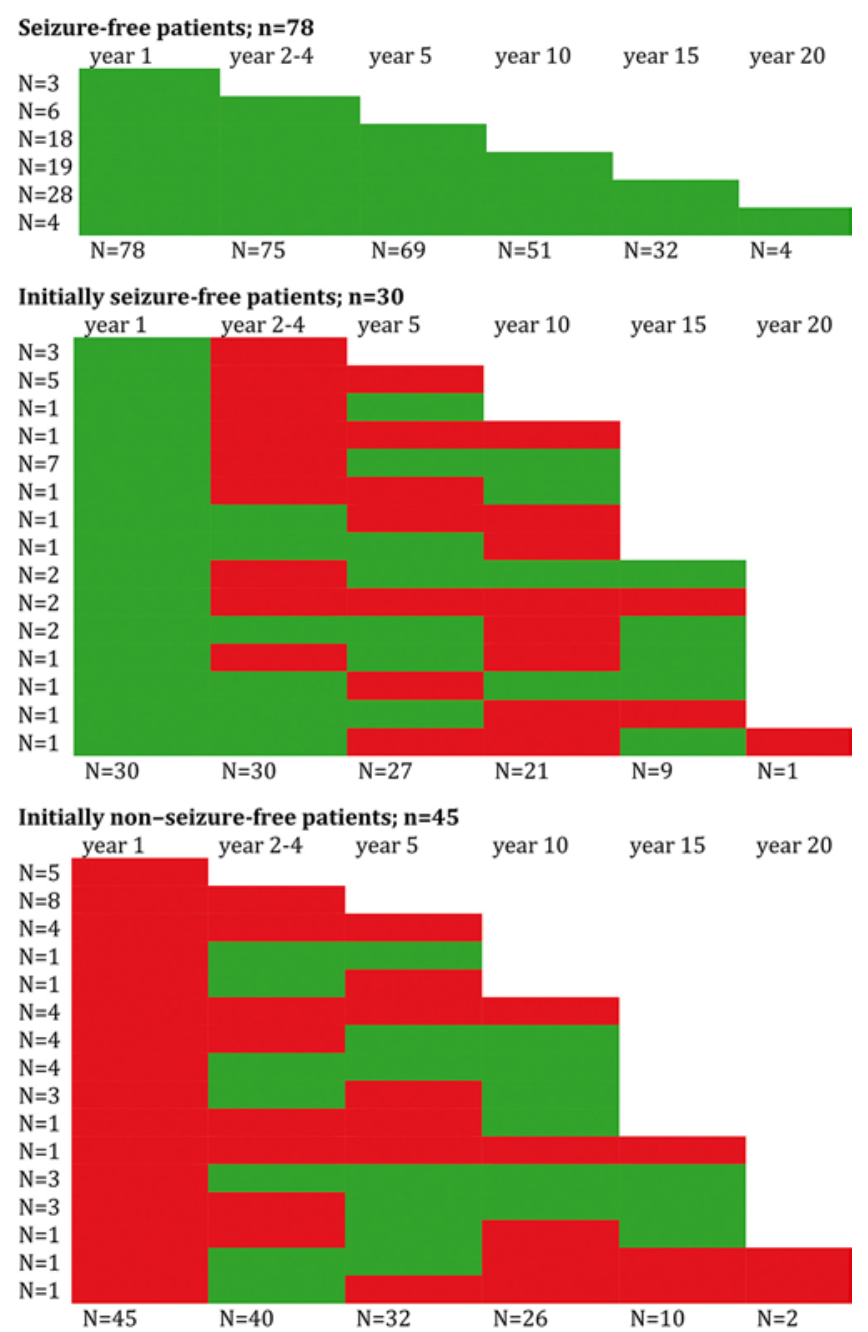

FIG. 1. Long-term postoperative outcome among 153 MTLE patients after SAHE according to ILAE Class 1. Figure is available in color online only. 


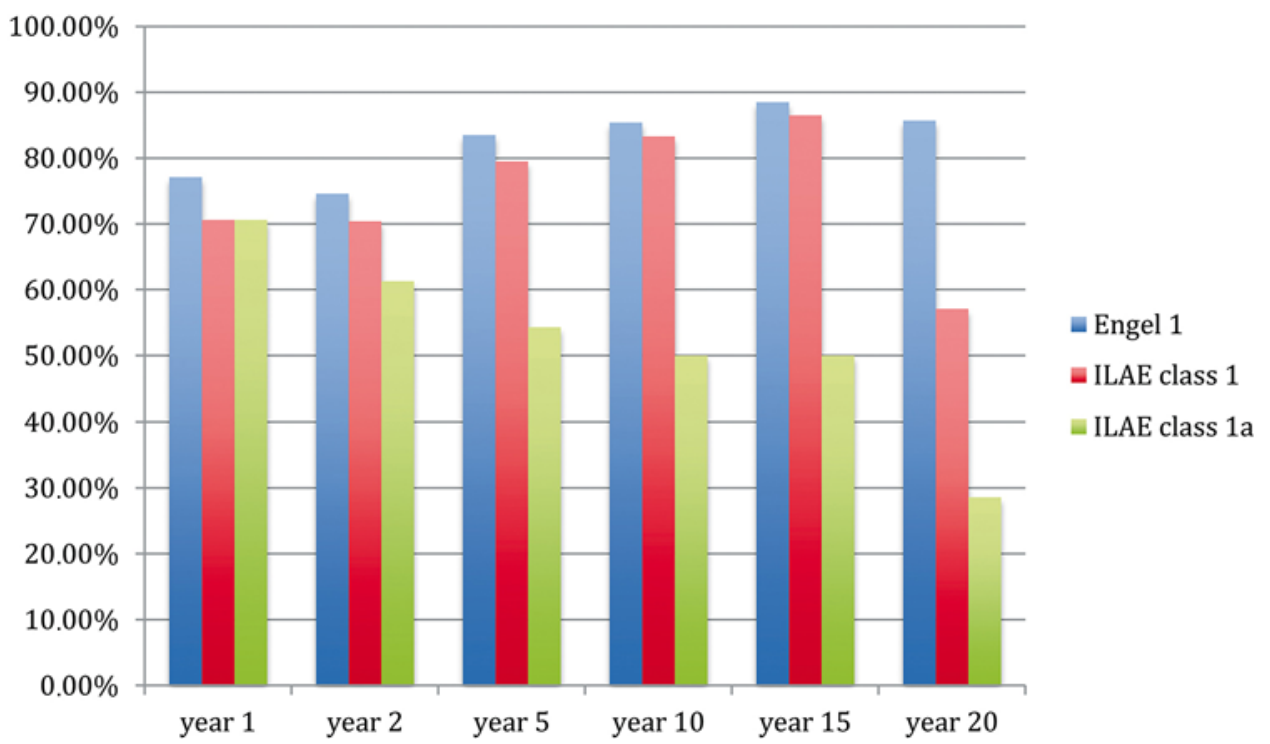

FIG. 2. Seizure-free outcomes of MTLE patients after SAHE according to 3 different classifications: Engel 1, free from disabling seizures; ILAE Class 1, no seizures during a 12-month period prior to assessment at 1, 2, 5, 10, 15, and 20 years postoperatively; ILAE Class 1a, absolute freedom from seizures and auras at any time after surgery. Figure is available in color online only.

\section{Temporal Lobectomy After Unsuccessful SAHE}

In 11 patients who were not seizure free after SAHE (ILAE Class 5 in 2 patients, ILAE Class 4 in 7 patients, ILAE Class 3 in 2 patients), reoperation via temporal lobectomy was performed after a mean period of 4.4 years from the SAHE (left side in 6 patients, right side in 5 patients). In none of these patients did histology reveal cortical dysplasia in the resected tissue. Magnetic resonance imaging after SAHE revealed residual mesiotemporal structures corresponding to the entorhinal and/or perirhinal cortex in 10 cases and residual hippocampal tissue in 3 cases. In none of these patients was selective resection of residual mesial structures alone intended since the potential for additional benefit in terms of seizure control due to the removal of neocortical tissue could not be dismissed preoperatively. In 4 of these patients (2 patients with ILAE Class 4 outcome, 2 patients with ILAE Class 5 outcome), a second operation was performed early, between 12 and 24 months after SAHE. The 7 remaining patients were temporarily free from seizures or had only rare disabling seizures (ILAE Classes 1-3), and 4 of these patients were completely seizure free between 5 months and 3.2 years after SAHE (ILAE Class 1a). Reoperation in these 7 patients was performed after a mean period of 8.0 years from SAHE. After a mean follow-up of 10.1 years from reoperation, $8(72.7 \%)$ of these 11 patients were seizure free (ILAE Class 1a in 4, ILAE Class 1 in 4). Among the 3 remaining patients, 1 had only auras (ILAE Class 2), 1 had rare seizures (ILAE Class 3), and 1 did not improve (ILAE Class 5). These data correspond to improvement to an Engel Class I outcome in $82 \%$ of the 11 patients.

\section{Overall Seizure Outcome}

After a mean follow-up of 10.0 years (median 10.1 years, range 12 months-20.1 years), 72 patients (47.0\%) were completely seizure free since (re)operation (ILAE Class 1a), 50 patients $(32.6 \%)$ were classified as ILAE
Class 1, 7 patients (4.6\%) as ILAE Class 2, 15 patients (9.8\%) as ILAE Class 3, 8 patients (5.2\%) as ILAE Class 4 , and 1 patient $(0.6 \%)$ as ILAE Class 5 . These data correspond to an Engel Class I outcome in $84.3 \%$ of the patients.

\section{Surgical Complications}

There were no deaths associated with surgery and no complications that resulted in a permanent neurological deficit. Complications with temporary morbidity were seen in 7 patients (4.6\%). Three of the patients needed surgery for a complication. In 2 of these patients, a hemispheric hygroma formation leading to intractable headaches required drainage through a bur hole 1 month and 5 months after SAHE, respectively. In the remaining patient, a hematoma in the resection cavity needed evacuation on the 2nd postoperative day due to progressive impairment of consciousness, which did not result in any permanent neurological compromise.

One patient had an infarct in the territory of the lateral posterior choroidal artery with severe hemiparesis that fully resolved 6 months after surgery. One patient had intermittent aphasia without any correlate on CT or followup MRI, although it had fully resolved by discharge on Day 12 after surgery. Transcranial Doppler ultrasonography of the middle cerebral artery was normal throughout that period. Two patients experienced temporary oculomotor nerve palsy.

No complications occurred in any of the patients who underwent reoperation.

\section{Discussion}

Although 2 recent meta-analyses of studies comparing selective medial temporal resections and standard ATL have noted a moderate advantage with the latter procedure, and despite the fact that an assumed reduction in the 
risk of selective surgery-related neuropsychological deterioration did not unequivocally stand the test of time, obtaining a good long-term seizure outcome with less extensive brain tissue resection remains an intuitively appealing concept. $5,17,26,27,31,52,56$ Our study provides information on long-term seizure outcome in a cohort of 153 consecutive adults with mesial temporal lobe epilepsy (MTLE) and hippocampal sclerosis on MRI who were selected for transsylvian SAHE at a single center, representing the largest consecutive series of patients treated in the MRI era with the most long-term follow-up information. ${ }^{59}$ After a median follow-up of 10 years, 78.4\% of the patients had a good SAHE outcome (Engel Class I). At 10 years, half of the patients had been completely seizure free since surgery (ILEA Class 1a or Engel Class IA).

Taking into account information available at the start of our epilepsy surgery program in $1994,15,16,21,44,50$ we decided to propose SAHE to all patients fulfilling electroclinical and radiological criteria for unilateral MTLE. Therefore, our practice does not allow for a comparison between the selective procedure and ATL, as the latter procedure was used only when the patient did not fulfill the diagnostic criteria for SAHE. In contrast, most investigators have discussed this selective surgery group of patients in comparison with patients assigned to ATL or patients with lesional MTLE including tumors and vascular malformation. ${ }^{23,24,41,52,59}$

While previous series have reported a lost-to-follow-up rate between $8.6 \%$ and $32.9 \%$, the referral pattern of our patients allowed for strict long-term observation with only 4 patients (2.5\%) lost to follow-up. 5,19,23,24,29,41,59,63 This also allowed for reoperation (ATL) in 11 cases of unsatisfactory seizure control or seizure recurrence. This strategy led to an excellent overall outcome (Engel Class I and ILEA Classes 1 and 2) in $84.3 \%$ of the patient population.

During the 20-year study period (1994-2014), we did not change our approach as we felt confirmed by our evolv-

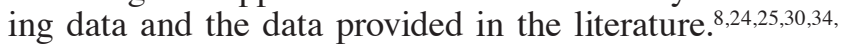
${ }^{52,59}$ Long-term seizure freedom rates at experienced centers were, indeed, similar between SAHE (54.4\%-80.4\%) and ATL (56\%-83\%). Recently, 2 meta-analyses of studies that compared epilepsy outcome between a selective and a standard approach pointed to a moderate advantage for the standard ATL. ${ }^{27,31}$ Methodological limitations of the included nonrandomized studies were addressed and comprise a lack of standardized follow-up, comparisons mostly with historical controls, and different SAHE techniques. Moreover, MRI findings and invasive recordings biased the choice of the surgical approach in some of the included studies. ${ }^{2,36}$

Beginning with Niemeyer in 1958, several authors have suggested that selective resection of the mesial temporal structures could be sufficient by having the same effect in terms of seizure outcome as a complete resection of the anterior temporal lobe. $38,40,61$

Regardless of the surgical corridor used to approach the medial temporal lobe, the different techniques somewhat incorrectly designated as "SAHE" aim to include the uncus and anterior parahippocampal gyrus into the resection volume.

We systematically used a transsylvian approach as de- veloped by Yaşargil ${ }^{62}$ and Wieser. ${ }^{60}$ In every single case we tried to maximize the amygdala resection and include all of the presumed entorhinal and perirhinal cortex into the resection volume, tailoring the resection to the individual sulcal pattern visualized on MRI. ${ }^{4,11,28,39}$

The failure of surgery to control seizures in MTLE can be discussed under various perspectives, differing in their practical relevance.,13,22 The question regarding the optimal extent of resection in the individual patient probably cannot be answered with absolute certainty prior to resection of the epileptogenic zone..$^{49}$ The critical involvement of the "temporopolar cortex" in a subgroup of patients with MTLE has been very well characterized based on stereoEEG (SEEG) investigations, ${ }^{1}$ with mostly the mediobasal parts of the pole showing early involvement and considered to be part of the epileptogenic zone. Subpially resecting the temporal pole medial to the dorsal temporopolar gyrus and down and laterally to the prolongation of the collateral sulcus would include the whole of the entorhinal and perirhinal transitional cortex in the resection. ${ }^{11,46}$ This region has been resected in the original descriptions of the transsylvian SAHE by a pterional craniotomy. ${ }^{3,62}$ Furthermore, postresection MRI studies, including a series by our group, have highlighted the importance of resecting this region with respect to seizure control, substantiating earlier observations. ${ }^{4,21,50,64}$ The variability of the sulcal anatomy in this basal temporopolar region must be taken into account, as certain anatomical variants of the temporobasal anatomy can misdirect the entorhinal resection during SAHE. ${ }^{11,39}$

While some authors reporting on SAHE deliberately do not include the parahippocampal gyrus in the resection, ${ }^{2}$ the postoperative MRI included in some reports has shown what we would consider an insufficient resection of anterior entorhinal and/or perirhinal cortex. ${ }^{37}$ As we have demonstrated in previous reports, however, residual entorhinal cortex represents the most important factor in SAHE failures. ${ }^{32,62}$ Working through a limited corticotomy without a retractor necessitates precise planning of the available angular range through this "cortical keyhole" to be able to access the structures to be resected. Lesions in the dorsal part of the anterior medial temporal lobe can be resected through a lateral subfrontal-transsylvian approach with supraorbital craniotomy. However, this approach does not allow for selective transamygdala resection of cortex partially lying under the sphenoid wing on the anterior middle fossa floor. ${ }^{17,37,47}$

Much emphasis has recently been put on the different white matter tracts transgressed or deliberately transected during the different approaches. ${ }^{9,12}$ The lateral transtemporal approaches, besides theoretically dissecting parallel to the optic radiation fibers and reducing the risk of a upper quadrant field defect, avoid injury to fiber tracts localized in the temporal stem in the roof of the ventricle. ${ }^{33,48}$ Although the uncinate fascicle can be preserved in the transsylvian approach, as evidenced by postoperative diffusion tensor imaging, ${ }^{61}$ we deliberately extended the resection to the upper margin of the amygdala, at least partially interrupting this fascicle as well as parts of the anterior commissure radiation. At this time we hypothesize that this disconnection is part of the procedure as 
there was a lack of evidence for relevant deficits related to it, but this aspect needs to be critically addressed in future studies. ${ }^{12,54}$ It seems more important to restrict the lateroposterior extension of the corticotomy along the inferior insular sulcus into the temporal stem to minimize the risk of interrupting the occipitofrontal fiber bundles that are an important component of the "ventral" language network. ${ }^{12}$

The lateral subtemporal SAHE approaches through either the parahippocampal or, more commonly, the fusiform gyrus, aiming at circumventing the optic radiation altogether, are interesting alternatives, with seizure outcomes reported in the same range as with the other techniques. ${ }^{10,25,34,42,53}$ Current difficulties in preoperatively assessing the course of the temporobasal veins, the angulation of the collateral sulcus changing from anterior vertical to posterior mediolateral-horizontal and hindering a strictly transsulcal access to the whole extent of the ventricle, as well as a steep working angle to access the upper amygdala-uncal region, can eventually lead to changing the intended corticotomy site to restrict damage to the inferior temporal gyri. ${ }^{53}$

In our experience, no permanent surgical morbidity has occurred after transsylvian SAHE, and we encountered no surgical complications in the 11 patients who had been treated with a second operation to complete the temporal resection. Minor complications were seen in only 7 patients $(4.6 \%)$ after SAHE. Similarly, other authors who compared the outcomes for transsylvian SAHE with those for ATL in MTLE did not report a difference in the complication profile. For instance, in the largest series reported by Clusmann et al., no difference in the complication profile among 3 resection types (ATL, transsylvian SAHE, lateral neocortical lesionectomy) was reported.$^{8}$

Even though there has been no clear-cut evidence that SAHE in MTLE provides a better seizure control rate than ATL over the study period, the fact that a tailored approach avoids a potentially unnecessary resection of neocortical

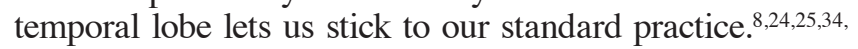
${ }^{52,59}$ In light of this, the aim of complete resection of the mesial structures (entorhinal cortex, amygdala, hippocampus, parahippocampus) eliminated the question of whether to perform a second resection limited to mesial structure remnants in cases of unfavorable seizure control. In our series, 114 patients $(74.5 \%)$ were seizure free (ILEA Class 1$)$ at a mean follow-up of 9.7 years after SAHE. In other words, $114(74.5 \%)$ of the 153 patients would have undergone resection of the anterior temporal lobe unnecessarily if we had followed the strategy of performing an upfront ATL. Only 11 patients $(7.2 \%)$ underwent reoperation to complete the resection to an ATL due to unsatisfactory seizure outcome, 8 of whom became completely seizure free. Thus, the overall seizure freedom rate of this tailored approach was $79.7 \%$ according to the ILAE classification and $83.6 \%$ according to the Engel classification, which is comparable to studies using upfront ATL.,29

In terms of the individual long-term seizure outcome, the rate of recurrence and late cure due to the "runningdown phenomenon" needs to be scrutinized. These rates were speculated to be more prominent in selective resections. As in the series by Wieser et al., we observed that a change from seizure freedom to the recurrence of seizures and vice versa could occur at any time after surgery. ${ }^{59}$ More importantly, however, this observation did not differ from data obtained after standard ATL in MTLE patients, arguing against the notion that seizure recurrence and late cure may be higher after SAHE.

\section{Conclusions}

We demonstrated a satisfactory long-term seizure outcome for transsylvian SAHE in a selected group of patients with refractory temporal lobe epilepsy. Having proved that the second-line strategy of an ATL (in the small percentage of patients whose SAHE failed) is highly effective, we think that we can reasonably propose SAHE to selected patients.

\section{References}

1. Barba C, Rheims S, Minotti L, Guénot M, Hoffmann D, Chabardès $\mathrm{S}$, et al: Temporal plus epilepsy is a major determinant of temporal lobe surgery failures. Brain 139:444451, 2016

2. Bate H, Eldridge P, Varma T, Wieshmann UC: The seizure outcome after amygdalohippocampectomy and temporal lobectomy. Eur J Neurol 14:90-94, 2007

3. Binder DK, Schramm J: Resective surgical techniques: mesial temporal lobe epilepsy, in Lüders HO: (ed): Textbook of Epilepsy Surgery. London: Informa, 2008, pp 1083-1093

4. Bonilha L, Yasuda CL, Rorden C, Li LM, Tedeschi H, de Oliveira E, et al: Does resection of the medial temporal lobe improve the outcome of temporal lobe epilepsy surgery? Epilepsia 48:571-578, 2007

5. Bujarski KA, Hirashima F, Roberts DW, Jobst BC, Gilbert KL, Roth RM, et al: Long-term seizure, cognitive, and psychiatric outcome following trans-middle temporal gyrus amygdalohippocampectomy and standard temporal lobectomy. J Neurosurg 119:16-23, 2013

6. Chabardès S, Kahane P, Minotti L, Tassi L, Grand S, Hoffmann D, et al: The temporopolar cortex plays a pivotal role in temporal lobe seizures. Brain 128:1818-1831, 2005

7. Chung MY, Walczak TS, Lewis DV, Dawson DV, Radtke R: Temporal lobectomy and independent bitemporal interictal activity: what degree of lateralization is sufficient? Epilepsia 32:195-201, 1991

8. Clusmann H, Schramm J, Kral T, Helmstaedter C, Ostertun B, Fimmers R, et al: Prognostic factors and outcome after different types of resection for temporal lobe epilepsy. J Neurosurg 97:1131-1141, 2002

9. Colnat-Coulbois S, Mok K, Klein D, Pénicaud S, Tanriverdi T, Olivier A: Tractography of the amygdala and hippocampus: anatomical study and application to selective amygdalohippocampectomy. J Neurosurg 113:1135-1143, 2010

10. Delev D, Wabbels B, Schramm J, Nelles M, Elger CE, von Lehe M, et al: Vision after trans-sylvian or temporobasal selective amygdalohippocampectomy: a prospective randomised trial. Acta Neurochir (Wien) 158:1757-1765, 2016

11. Ding SL, Van Hoesen GW, Cassell MD, Poremba A: Parcellation of human temporal polar cortex: a combined analysis of multiple cytoarchitectonic, chemoarchitectonic, and pathological markers. J Comp Neurol 514:595-623, 2009

12. Duffau H, Thiebaut de Schotten M, Mandonnet E: White matter functional connectivity as an additional landmark for dominant temporal lobectomy. J Neurol Neurosurg Psychiatry 79:492-495, 2008

13. Engel J Jr: When is temporal lobe epilepsy not temporal lobe epilepsy? Brain 139:309-312, 2016 (Letter)

14. Engel J Jr, McDermott MP, Wiebe S, Langfitt JT, Stern JM, Dewar S, et al: Early surgical therapy for drug-resistant tem- 
poral lobe epilepsy: a randomized trial. JAMA 307:922-930, 2012

15. Engel J Jr, Van Ness PC, Rasmussen TB, Ojemann LM: Outcome with respect to epileptic seizures, in Engel J Jr (ed): Surgical Treatment of the Epilepsies, ed 2. New York: Raven Press, 1993, pp 609-621

16. Feindel W, Rasmussen T: Temporal lobectomy with amygdalectomy and minimal hippocampal resection: review of 100 cases. Can J Neurol Sci 18 (4 Suppl):603-605, 1991

17. Figueiredo EG, Deshmukh P, Nakaji P, Crusius MU, Teixeira MJ, Spetzler RF, et al: Anterior selective amygdalohippocampectomy: technical description and microsurgical anatomy. Neurosurgery 66 (3 Suppl Operative):45-53, 2010

18. Foldvary N, Klem G, Hammel J, Bingaman W, Najm I, Lüders H: The localizing value of ictal EEG in focal epilepsy. Neurology 57:2022-2028, 2001

19. Foldvary N, Nashold B, Mascha E, Thompson EA, Lee N, McNamara JO, et al: Seizure outcome after temporal lobectomy for temporal lobe epilepsy: a Kaplan-Meier survival analysis. Neurology 54:630-634, 2000

20. Gil-Nagel A, Risinger MW: Ictal semiology in hippocampal versus extrahippocampal temporal lobe epilepsy. Brain 120:183-192, 1997

21. Goldring S, Edwards I, Harding GW, Bernardo KL: Results of anterior temporal lobectomy that spares the amygdala in patients with complex partial seizures. J Neurosurg 77:185193, 1992

22. Grote A, Witt JA, Surges R, von Lehe M, Pieper M, Elger $\mathrm{CE}$, et al: A second chance-reoperation in patients with failed surgery for intractable epilepsy: long-term outcome, neuropsychology and complications. J Neurol Neurosurg Psychiatry 87:379-385, 2016

23. Hardy SG, Miller JW, Holmes MD, Born DE, Ojemann GA, Dodrill CB, et al: Factors predicting outcome of surgery for intractable epilepsy with pathologically verified mesial temporal sclerosis. Epilepsia 44:565-568, 2003

24. Hemb M, Palmini A, Paglioli E, Paglioli EB, Costa da Costa J, Azambuja N, et al: An 18-year follow-up of seizure outcome after surgery for temporal lobe epilepsy and hippocampal sclerosis. J Neurol Neurosurg Psychiatry 84:800-805, 2013

25. Hori T, Tabuchi S, Kurosaki M, Kondo S, Takenobu A, Watanabe T: Subtemporal amygdalohippocampectomy for treating medically intractable temporal lobe epilepsy. Neurosurgery 33:50-57, 1993

26. Hoyt AT, Smith KA: Selective amygdalohippocampectomy. Neurosurg Clin N Am 27:1-17, 2016

27. Hu WH, Zhang C, Zhang K, Meng FG, Chen N, Zhang JG: Selective amygdalohippocampectomy versus anterior temporal lobectomy in the management of mesial temporal lobe epilepsy: a meta-analysis of comparative studies. J Neurosurg 119:1089-1097, 2013

28. Huntgeburth SC, Petrides M: Morphological patterns of the collateral sulcus in the human brain. Eur J Neurosci 35:1295-1311, 2012

29. Janszky J, Janszky I, Schulz R, Hoppe M, Behne F, Pannek HW, et al: Temporal lobe epilepsy with hippocampal sclerosis: predictors for long-term surgical outcome. Brain 128:395-404, 2005

30. Jeong SW, Lee SK, Hong KS, Kim KK, Chung CK, Kim H: Prognostic factors for the surgery for mesial temporal lobe epilepsy: longitudinal analysis. Epilepsia 46:1273-1279, 2005

31. Josephson CB, Dykeman J, Fiest KM, Liu X, Sadler RM, Jette N, et al: Systematic review and meta-analysis of standard vs selective temporal lobe epilepsy surgery. Neurology 80:1669-1676, 2013

32. Jung R, Aull-Watschinger S, Moser D, Czech T, Baumgartner $\mathrm{C}$, Bonelli-Nauer $\mathrm{S}$, et al: Is reoperation an option for patients with temporal lobe epilepsy after failure of surgery? Seizure 22:502-506, 2013

33. Kier EL, Staib LH, Davis LM, Bronen RA: MR imaging of the temporal stem: anatomic dissection tractography of the uncinate fasciculus, inferior occipitofrontal fasciculus, and Meyer's loop of the optic radiation. AJNR Am J Neuroradiol 25:677-691, 2004

34. Little AS, Smith KA, Kirlin K, Baxter LC, Chung S, Maganti $\mathrm{R}$, et al: Modifications to the subtemporal selective amygdalohippocampectomy using a minimal-access technique: seizure and neuropsychological outcomes. J Neurosurg 111:1263-1274, 2009

35. Loddenkemper T, Kotagal P: Lateralizing signs during seizures in focal epilepsy. Epilepsy Behav 7:1-17, 2005

36. Mackenzie RA, Matheson J, Ellis M, Klamus J: Selective versus non-selective temporal lobe surgery for epilepsy. J Clin Neurosci 4:152-154, 1997

37. Martens T, Merkel M, Holst B, Brückner K, Lindenau M, Stodieck S, et al: Vascular events after transsylvian selective amygdalohippocampectomy and impact on epilepsy outcome. Epilepsia 55:763-769, 2014

38. Niemeyer P: The transventricular amygdala-hippocampectomy in temporal lobe epilepsy, in Baldwin M, Bailey P (eds): Temporal Lobe Epilepsy. Springfield, IL: Charles C Thomas, 1958, pp 461-482

39. Novak K, Czech T, Prayer D, Dietrich W, Serles W, Lehr S, et al: Individual variations in the sulcal anatomy of the basal temporal lobe and its relevance for epilepsy surgery: an anatomical study performed using magnetic resonance imaging. J Neurosurg 96:464-473, 2002

40. Olivier A: Transcortical selective amygdalohippocampectomy in temporal lobe epilepsy. Can J Neurol Sci 27 (Suppl 1):S68-S76, S92-S96, 2000

41. Ozkara C, Uzan M, Benbir G, Yeni N, Oz B, Hanoğlu L, et al: Surgical outcome of patients with mesial temporal lobe epilepsy related to hippocampal sclerosis. Epilepsia 49:696699, 2008

42. Park TS, Bourgeois BF, Silbergeld DL, Dodson WE: Subtemporal transparahippocampal amygdalohippocampectomy for surgical treatment of mesial temporal lobe epilepsy. Technical note. J Neurosurg 85:1172-1176, 1996

43. Pataraia E, Lurger S, Serles W, Lindinger G, Aull S, Leutmezer F, et al: Ictal scalp EEG in unilateral mesial temporal lobe epilepsy. Epilepsia 39:608-614, 1998

44. Penfield W, Baldwin M: Temporal lobe seizures and the technic of subtotal temporal lobectomy. Ann Surg 136:625-634, 1952

45. Pfänder M, Arnold S, Henkel A, Weil S, Werhahn KJ, Eisensehr I, et al: Clinical features and EEG findings differentiating mesial from neocortical temporal lobe epilepsy. Epileptic Disord 4:189-195, 2002

46. Pruessner JC, Köhler S, Crane J, Pruessner M, Lord C, Byrne A, et al: Volumetry of temporopolar, perirhinal, entorhinal and parahippocampal cortex from high-resolution MR images: considering the variability of the collateral sulcus. Cereb Cortex 12:1342-1353, 2002

47. Reisch R, Stadie A, Kockro R, Gawish I, Schwandt E, Hopf $\mathrm{N}$ : The minimally invasive supraorbital subfrontal key-hole approach for surgical treatment of temporomesial lesions of the dominant hemisphere. Minim Invasive Neurosurg 52:163-169, 2009

48. Ribas EC, Yagmurlu K, Wen HT, Rhoton AL Jr: Microsurgical anatomy of the inferior limiting insular sulcus and the temporal stem. J Neurosurg 122:1263-1273, 2015

49. Schramm J: Temporal lobe epilepsy surgery and the quest for optimal extent of resection: a review. Epilepsia 49:12961307,2008

50. Siegel AM, Wieser HG, Wichmann W, Yasargil GM: Relationships between MR-imaged total amount of tissue 
removed, resection scores of specific mediobasal limbic subcompartments and clinical outcome following selective amygdalohippocampectomy. Epilepsy Res 6:56-65, 1990

51. Steinhoff BJ, So NK, Lim S, Lüders HO: Ictal scalp EEG in temporal lobe epilepsy with unitemporal versus bitemporal interictal epileptiform discharges. Neurology 45:889-896, 1995

52. Tanriverdi T, Olivier A, Poulin N, Andermann F, Dubeau F: Long-term seizure outcome after mesial temporal lobe epilepsy surgery: corticalamygdalohippocampectomy versus selective amygdalohippocampectomy. J Neurosurg 108:517524, 2008

53. Thudium MO, Campos AR, Urbach H, Clusmann H: The basal temporal approach for mesial temporal surgery: sparing the Meyer loop with navigated diffusion tensor tractography. Neurosurgery 67 (2 Suppl Operative):385-390, 2010

54. Von Der Heide RJ, Skipper LM, Klobusicky E, Olson IR: Dissecting the uncinate fasciculus: disorders, controversies and a hypothesis. Brain 136:1692-1707, 2013

55. Wellmer J, Quesada CM, Rothe L, Elger CE, Bien CG, Urbach H: Proposal for a magnetic resonance imaging protocol for the detection of epileptogenic lesions at early outpatient stages. Epilepsia 54:1977-1987, 2013

56. Wendling AS, Steinhoff BJ, Bodin F, Staack AM, Zentner J, Scholly J, et al: Selective amygdalohippocampectomy versus standard temporal lobectomy in patients with mesiotemporal lobe epilepsy and unilateral hippocampal sclerosis: postoperative facial emotion recognition abilities. Epilepsy Res 111:26-32, 2015

57. Wiebe S, Blume WT, Girvin JP, Eliasziw M: A randomized, controlled trial of surgery for temporal-lobe epilepsy. N Engl J Med 345:311-318, 2001

58. Wieser HG, Blume WT, Fish D, Goldensohn E, Hufnagel A, King D, et al: ILAE Commission Report. Proposal for a new classification of outcome with respect to epileptic seizures following epilepsy surgery. Epilepsia 42:282-286, 2001

59. Wieser HG, Ortega M, Friedman A, Yonekawa Y: Long-term seizure outcomes following amygdalohippocampectomy. J Neurosurg 98:751-763, 2003

60. Wieser HG, Yaşargil MG: Selective amygdalohippocampectomy as a surgical treatment of mesiobasal limbic epilepsy. Surg Neurol 17:445-457, 1982
61. Yaşargil MG, Krayenbühl N, Roth P, Hsu SP, Yaşargil DC: The selective amygdalohippocampectomy for intractable temporal limbic seizures. J Neurosurg 112:168-185, 2010

62. Yaşargil MG, Teddy PJ, Roth P: Selective amygdalo-hippocampectomy. Operative anatomy and surgical technique. Adv Tech Stand Neurosurg 12:93-123, 1985

63. Yoon HH, Kwon HL, Mattson RH, Spencer DD, Spencer SS: Long-term seizure outcome in patients initially seizure-free after resective epilepsy surgery. Neurology 61:445-450, 2003

64. Zachenhofer I, Novak K, Baumgartner C, Prayer D, Czech T: Reoperation after selective amygdalohippocampectomy: an MRI analysis of the extent of temporomesial resection in ten cases. Acta Neurochir (Wien) 153:239-248, 2011

\section{Disclosures}

The authors report no conflict of interest concerning the materials or methods used in this study or the findings specified in this paper.

\section{Author Contributions}

Conception and design: Czech, Dorfer, Baumgartner, Pataraia. Acquisition of data: Czech, Dorfer, Aull-Watschinger, Baumgartner, Jung, Kasprian, Pirker, Seidl, Stefanits, Trimmel, Pataraia. Analysis and interpretation of data: Czech, Dorfer, Baumgartner, Pataraia. Drafting the article: Czech, Dorfer. Critically revising the article: Czech, Dorfer, Baumgartner, Novak, Pataraia. Reviewed submitted version of manuscript: Czech, Dorfer, Baumgartner, Novak, Pataraia. Approved the final version of the manuscript on behalf of all authors: Czech. Administrative/ technical/material support: Dorfer, Pataraia. Study supervision: Czech, Pataraia.

\section{Correspondence}

Thomas Czech, Department of Neurosurgery, Medical University of Vienna, Währinger Gürtel 18-20, Vienna 1090, Austria. email: thomas.czech@meduniwien.ac.at. 